\title{
Thermodynamics-Acoustics Coupling Studies on Self-excited Combustion Oscillations Maximum Growth Rate
}

\author{
Dan Zhao*
}

Department of Mechanical Engineering, College of Engineering, University of Canterbury, Private Bag 4800, Christchurch 8041, New Zealand

(C) Science Press, Institute of Engineering Thermophysics, CAS and Springer-Verlag GmbH Germany, part of Springer Nature 2020

\begin{abstract}
Unlike electric vehicles and electric aircrafts, hydrocarbon-fuelled (fossil) engine systems are much noisier. By conducting one-step chemical reaction-thermodynamics-acoustics coupling studies and experimental measurements, we explore the universal physics of how hydrocarbon-fuelled combustion is a noise maker. We also explain that how combustion-sustained noise at a particular frequency $\omega$ is intrinsically selected. These frequencies correspond to the acoustic resonance nature of the combustor. We find that a reacting gas in which the rate of chemical reacting increases with temperature is intrinsically and naturally unstable with respect to acoustic wave motion, since its modal growth rate $\alpha$ is greater than 0 . Acoustic disturbances tend to exponentially i.e. $\exp (\alpha t)$ increased first and then are limited by nonlinear effects and finally grow into limit cycle oscillations. The growth rate $\alpha$ is found to increase first and then decrease with the gradient of the heat release rate with respect to the temperature change, i.e. heat capacity. The maximum $(\alpha / \omega)_{\max }$ depends on the specific heat ratio $\gamma$, which is related to the speed of sound. The unstable nature could be changed by introducing some acoustic dissipative/damping mechanism, such as the boundary layer viscous drag and boundary losses. It is shown that such losses could lead to increased critical heat capacity, below which stable combustors can be designed. Finally, the acoustical energy consisting of both potential and kinetic energy is found to grow exponentially faster by $100 \%$ than the acoustic disturbance amplitude.
\end{abstract}

Keywords: Thermodynamics, Acoustics, Hydrocarbon, Chemical Reaction, Energy Conversion, Thermo-acoustics

\section{Introduction}

Self-excited thermo-acoustic instability $[1,2]$ is an interdisciplinary phenomena. It typically leads to large-amplitude pulsating oscillations of thermodynamic properties, such as pressure, temperature and velocity [2]. Over the past few decades, how to design a stable combustion system in the absence of such self-sustained pulsations [3] is a major challenge for power generation [4], aeronautics $[5,6]$, and aerospace propulsion $[7,8]$ industrial sectors. Thermo-acoustic instability occurs due to the 'constructive' coupling/loop between acoustics and unsteady heat release from a heat source (typically either a flame or an electrical heater) [9]. The feedback loop between unsteady heat release and acoustics is physically explained by Rayleigh criterion [2, 10]. It reveals how thermo-acoustic energy conversion will determine whether acoustic modes are augmented or damped by unsteady combustion, as well as altering the frequency and amplitude. When such instability occurs, 
large-amplitude pulsating oscillations may lead to a dramatic loss of the system performance, and even catastrophic failures, due to the flame flashback, overheating or structural vibration. The key challenge of understanding and predicting such interdisciplinary thermo-acoustics physics involved in the 'interaction': acoustics, single-/multiple-phase flows, hydrocarbon-air mixing [11], vortex/turbulence dynamics [12], thermodynamics, and chemical reactions.

A hydrocarbon-fuelled flame confined in a combustor is a monopole sound source. Unsteady heat release by burning such hydrocarbon is identified as the dominant and active contributor to acoustical energy gain [13]. The combustor is typically acoustically resonant in nature. Thus predicting eigen-frequencies and mode-shapes and the growth rate of combustion-driven acoustic disturbances is needed [14]. Determining the modeshapes and eigen-frequencies [15] is not a difficult task by solving linearized Euler [16] or Helmholtz solvers in $1 \mathrm{D}$ network [17, 18] or complex 2D [19] and 3D models [20]. However, estimating the growth rate [21] in real time is quite challenging [22], since it requires more accurate online evaluation of 1) boundaries conditions, 2) the flame response (flame transfer/describing function for example) and 3) intrinsic acoustic dissipation [23] due to acoustics-vorticity interaction and wall friction. Conventional LEE and Helmholtz solvers cannot be applied to obtain real-time growth rate. However, the determination of the growth rate plays a critical role in the design and safe operation of a power generation or propulsion system. In addition, the design of a robust acoustic damper [24] to be applied in combustors needs the knowledge of the thermos-acoustics growth rate [25]. Noiray and Denisov [25] proposed a Fokker-Planckbased method to identify the growth rate from the experimentally measured pressure data. It is indeed important to the stability map [18] the combustor is operated so that a small change in operating condition does not trigger thermoacoustic instability [26].

Analytical approaches to predict thermos-acoustics eigen-modes are applicable. The main challenge is how to theoretically model the unsteady heat release from the dynamic flame. There are three conventional approaches. One is to use the classical $G$-equation describing the flame dynamic response [27] to acoustic disturbance or to use Crocco model (also known n- $\tau$ ) $[17,28]$. The other is to use FTF (Flame Transfer Function) [21, 25] or FDF (Flame Describing Function) [29] to describe the linear and nonlinear flame responses. In practice, the flame responses need to be laser-diagnostically and quantitatively measured. The last but the least method is to numerically describe the flame response in $2 \mathrm{D} / 3 \mathrm{D}$ RANS (Reynolds-Averaged Navier-Stokes), LES (Large Eddy Simulations) or DNS (Direct Numerical
Simulations) studies, for example turbulence combustion is captured by using Moment methods for reactive scalars, Eddy Dissipation Concept (EDC), or flamelet models. These numerical approaches in time domain are computational costly and need detailed experimental data for calibration.

To the best knowledge of the author, there is no unified theory in the literature to explain the physics behind self-excited combustion noise resulting from chemical reaction-thermodynamics-acoustics coupling. In addition, there is a lack of prediction on the growth rate of acoustic disturbances in a heated thermal-fluids system. In this work, fundamental chemical reactionthermodynamics-acoustics coupling studies are conducted to shed lights on predicting how the acoustic disturbances and acoustical energy [30] evolve, and how the combustion-driven noise frequency $\omega(\mathrm{rad} / \mathrm{s})$ is naturally selected by the acoustic resonant nature of the combustor. The effect of acoustic losses/damping characterizing the boundary losses and viscous boundary layer is also evaluated by varying the heat capacity. The predicted maximum growth rate $\alpha$ of the acoustic disturbances is validated by the experimental data obtained from a well-designed T-shaped combustor with a cooling flow perforated pipe. It is found that the normalized maximum growth rate $G_{\max } \equiv(\alpha / \omega)_{\max }$ is $9.2 \times$ $10^{-2} \mathrm{rad} / \mathrm{s}^{2}$. Finally, the self-excited combustion noise is shown to correspond to the longitudinal and/or azimuthal modes of the acoustic resonant nature of the combustor.

\section{Generation Mechanism of Combustion-excited Acoustics}

\subsection{Chemical Reaction-Thermodynamics-Acoustics Analysis}

If the mixture of the hydrocarbon $\left(\mathrm{C}_{\mathrm{x}} \mathrm{H}_{\mathrm{y}}\right)$ fuel and air used in a given engine system under consideration is assumed to follow the perfect gas law as

$$
p=\rho T R=\rho T \frac{R_{u}}{M W}
$$

where $p$ denotes the pressure, $T$ is the temperature and $\rho$ is the mixture density. $R$ is the gas constant per gram of the gas mixture. $R_{u}=8.314 \mathrm{~J} /(\mathrm{mol} \cdot \mathrm{K})$ is the universal gas constant. $M W$ is the mixture molecular weight of $\mathrm{C}_{\mathrm{x}} \mathrm{H}_{\mathrm{y}}$.

For hydrocarbon-fuelled combustion with air as an oxidiser, it can be shown that

$$
\begin{aligned}
\mathrm{C}_{x} \mathrm{H}_{y}+ & \left(x+\frac{y}{4}\right)\left(\mathrm{O}_{2}+3.76 \mathrm{~N}_{2}\right) \rightarrow \\
& x \mathrm{CO}_{2}+\frac{y}{2} \mathrm{H}_{2} \mathrm{O}+3.76\left(x+\frac{y}{4}\right) \mathrm{N}_{2}
\end{aligned}
$$

where nitrogen $\mathrm{N}_{2}$ is assumed to not involve in chemical combustion for simplicity. Furthermore, the 
combustion rate is assumed to be infinite fast (i.e. one-step chemical reaction) and no turbulence eddies are assumed to present in the combustor. If $\mathcal{H}$ denotes the energy released per kilo gram of the mixture by the complete reaction, then the heat release rate $Q(T)$ is given as

$$
Q(T)=-\frac{H_{\mathrm{C}_{x} \mathrm{H}_{y}}}{n_{\mathrm{C}_{x} \mathrm{H}_{y}}(t=0)} \frac{\partial}{\partial t}\left(n_{\mathrm{C}_{x} \mathrm{H}_{y}}\right)
$$

where $n_{\mathrm{C}_{x} \mathrm{H}_{y}}$ denotes the kilo mole number of the species $\mathrm{C}_{x} \mathrm{H}_{y}$ per kilo gram of the mixture at time $t$. $n_{\mathrm{C}_{x} \mathrm{H}_{y}}(t=0)$ denotes the number of moles at $t=0 \mathrm{~s}$. As far as Eq. (2) is concerned, the rate of the chemical reaction of $n_{\mathrm{C}_{x} \mathrm{H}_{y}}$ is given as

$$
\frac{\partial}{\partial t}\left(n_{\mathrm{C}_{x} \mathrm{H}_{y}}\right)=\left(x+\frac{y}{4}\right) \frac{\partial}{\partial t}\left(n_{\mathrm{O}_{2}}\right)=-\kappa n_{\mathrm{C}_{x} \mathrm{H}_{y}} n_{\mathrm{O}_{2}}^{\left(x+\frac{y}{4}\right)}
$$

$\kappa$ is the Arrhenius reaction rate and it is given as

$$
\kappa=C_{f} e^{-E_{a} / T k_{B}}
$$

where $C_{\mathrm{f}}$ is the frequency factor of molecule collision, $E_{\mathrm{a}}$ is the activation energy. $k_{B}$ is the Boltzmann constant. Thus the heat release $Q(T)$ is given as

$$
Q(T)=\frac{H_{\mathrm{C}_{x} \mathrm{H}_{y}} C_{f} e^{-E_{a} / T k_{B}}}{n_{\mathrm{C}_{x} \mathrm{H}_{y}}(t=0)} n_{\mathrm{C}_{x} \mathrm{H}_{y}} n_{\mathrm{O}_{2}}^{\left(x+\frac{y}{4}\right)}
$$

It is worth noting that Eqs.(2)-(5) could also be applied to characterize the one-step combustion of hydrogen $\left(\mathrm{H}_{2}\right)$, as $x=0$ and $y=2$.

If $U(\mathrm{~T})$ denote the internal energy [50] per $\mathrm{kg}$ of the mixture of $\mathrm{C}_{x} \mathrm{H}_{y}$ and air, then it can be shown that

$$
U(T)=c_{v} T=\frac{p}{\rho(\gamma-1)}
$$

where $\gamma$ is the specific heat ratio, $c_{\mathrm{V}}$ is the specific heat at constant volume. By applying the first law of thermodynamics, the heat release $Q(T)$ at constant pressure condition is following

$$
\frac{\mathrm{d} U}{\mathrm{~d} t}+p \frac{\mathrm{d}(1 / \rho)}{\mathrm{d} t}=Q(T)
$$

The fuel-air mixture properties are assumed to consist of the mean value denoted with overbar and fluctuation part as

$$
\begin{aligned}
& \frac{\rho}{\bar{\rho}}=1+\psi+\vartheta\left(\psi^{2}\right) \\
& \frac{T}{\bar{T}}=1+\Gamma+\vartheta\left(T^{2}\right) \\
& \frac{p}{\bar{p}}=1+P+\vartheta\left(P^{2}\right)
\end{aligned}
$$

By keeping the term of $\psi$ and $\Gamma$ while neglecting the high-order terms, substituting Eqs. (9a) and (9b) into Eq. (8) gives

$$
\frac{\mathrm{d}}{\mathrm{d} t} \ln \left\{\frac{(1+\Gamma)}{(1+\psi)^{\gamma-1}}\right\}=\frac{(\gamma-1)}{(1+\Gamma)} \frac{Q(T)}{R \bar{T}}
$$

The mass and momentum conservation holds as

$$
\begin{aligned}
& \nabla \cdot(p \boldsymbol{u})+\partial \rho / \partial t=0 \\
& \frac{\mathrm{d} \boldsymbol{u}}{\mathrm{d} t}+\zeta \boldsymbol{u}+\frac{1}{\rho} \nabla p=0
\end{aligned}
$$

The vector $\boldsymbol{u}$ denotes the working gas velocity and it's time derivative is given as

$$
\begin{aligned}
\frac{\mathrm{d}}{\mathrm{d} t} \boldsymbol{u} & =\frac{\partial \boldsymbol{u}}{\partial t}+(\boldsymbol{u} \cdot \nabla) \boldsymbol{u} \\
& =\frac{\partial}{\partial t} \boldsymbol{u}+\nabla\left(\frac{1}{2} \boldsymbol{u}^{2}\right)-\boldsymbol{u} \times \nabla \times \boldsymbol{u}
\end{aligned}
$$

To generalize our analysis, we introduce velocity potential $\Phi$ as $\boldsymbol{u}=-\nabla \Phi$. Eqs. (11a) and (11b) can be shown that

$$
\begin{gathered}
\frac{\mathrm{d}}{\mathrm{d} t} \ln (1+\psi)=\nabla^{2} \phi \\
\frac{\partial}{\partial t} \nabla \phi-\nabla\left(\frac{1}{2} u^{2}\right)=R \nabla T+\frac{R T}{\rho} \nabla \rho
\end{gathered}
$$

Eq. (10) and Eqs. (13a) and (13b) can be further simplified by using Eqs. (9a) and (9b) and neglecting the $2^{\text {nd }}$-order power terms of $\psi$ and $\Gamma$ as given as

$$
\begin{gathered}
\frac{\partial}{\partial t}(\Gamma+(1-\gamma) \psi)=\frac{\gamma-1}{R \bar{T}} Q(T) \\
\partial \phi / \partial t=R \bar{T}(\Gamma+\psi) \\
\partial \psi / \partial t=\nabla^{2} \phi
\end{gathered}
$$

It is worth noting that Eq. (14b) is obtained by using the linearized Eq. (13b) and conducting integration and neglecting the terms involved with the time dependence instead of space dependence.

The classical heat-driven acoustic wave equation is obtained by differentiating Eq. (14b) with time $t$ and applying Eqs. (14a) and (14c) as $\frac{\partial^{2} \Phi}{\partial t^{2}}-\gamma R \bar{T} \nabla^{2} \Phi=$ $(\gamma-1) Q(T)$. When acoustic losses/damping at the combustion inlet and outlet, and the viscous boundary layer near the inner combustor surface is considered, a more generalized heat-driven acoustic equation is given as

$$
\frac{\partial^{2}}{\partial t^{2}} \phi+\zeta \frac{\partial}{\partial t} \phi-\gamma R \bar{T} \nabla^{2} \phi=(\gamma-1) Q(T)
$$

The second term on the LHS (left-hand-side) of Eq. (15) involving $\zeta$ characterize the overall acoustic losses. Here $\gamma R \bar{T}$ is related to the speed of sound $c_{\mathrm{s}}$ as $c_{z}^{2}=\gamma R \bar{T}$. In practical combustors, acoustic damping/ losses are present and associated with the viscous boundary layer and the combustor inlet and outlet 
acoustic impedance change.

Differentiating Eq. (15) with respect to time $t$ give rises to

$$
\begin{aligned}
& \frac{\partial^{3}}{\partial t^{3}} \phi+\zeta \frac{\partial^{2}}{\partial t^{2}} \phi-c_{s}^{2} \frac{\partial}{\partial t}\left(\nabla^{2} \phi\right) \\
= & (\gamma-1) \bar{T} \frac{\mathrm{d} Q}{\mathrm{~d} T} \frac{\mathrm{d} \Gamma}{\mathrm{d} t} \\
= & R \bar{T} \delta Q_{T} \frac{\partial \Gamma}{\partial t}
\end{aligned}
$$

where $\delta Q_{T}=\frac{\gamma-1}{R}\left(\frac{\mathrm{d} Q}{\mathrm{~d} T}\right)_{\bar{T}}$. In physics, it denotes

the heat capacity characterizing the heat release rate with temperature increased.

By manipulating Eqs. (14a) and (14c), the following relation between $\Gamma$ and $\Phi$ holds as

$$
\frac{\partial}{\partial t} \Gamma=\frac{1}{R \bar{T}} \frac{\partial^{2}}{\partial t^{2}} \phi-\nabla^{2} \phi
$$

Thus Eq. (16) could be rewritten into

$$
\frac{\partial^{3}}{\partial t^{3}} \phi+\zeta \frac{\partial^{2}}{\partial t^{2}} \phi-c_{S}^{2} \frac{\partial}{\partial t} \nabla^{2} \phi=\delta Q_{T}\left\{\frac{\partial^{2}}{\partial t^{2}} \phi-\frac{c_{S}^{2}}{\gamma} \nabla^{2} \phi\right\}
$$

Eq. (18) describes in physics how acoustic disturbances are generated and propagate in the combustor. It also reveals the energy conversion between thermal energy $\delta Q_{T}$ and the acoustical energy $W_{\text {a }}$, which is defined and determined in the following section.

Now let's examine the acoustic disturbance characterized by $\Phi$ in Eq. (18). The acoustic disturbances may decay or grow from initial amplitude over time. It would be a general practice to assume that

$$
\begin{aligned}
\phi(r, \theta, z, t) & =\phi_{0} e^{(\alpha+j \omega) t} F(r, \theta, z) \\
& =\phi_{0} e^{(\alpha+j \omega) t} \sum_{n=1}^{\infty} \sum_{l=1}^{\infty} B(r) e^{\left(j n \theta+j z k_{z l}\right)}
\end{aligned}
$$

where $\Phi_{0}$ denotes the initial amplitude of the acoustic disturbance present in the combustion system at $t=0 \mathrm{~s}$. $\alpha$ is a real value, and $\alpha$ denotes the growth $(\alpha>0)$ or decay $(\alpha<0)$ rate of the acoustic disturbances. In the literature, $\mathrm{Su}$ et al. [31] conducted experimental measurements of the decay rate (characterizing the acoustic attenuation/loss effect) of a solid-propellant rocket motor. The decay rate $G=\alpha / \omega$ is in the range of $-0.05 \leq G \leq-0.0068$ [31]. When $\alpha=0$, the combustion system is marginally stable, since the acoustic disturbances is neither amplified nor decayed with time.

\subsection{Acoustic Resonance Nature of the Combustor}

Since most of practical gas turbines, aero-engines and rocket motors may simplified to be can- or annular-type combustors. It is interesting to shed light on the acoustic nature (such as resonance frequency) of these combustors. In Eq. (19), $\omega$ denotes the frequency of the acoustic perturbations in the combustion system. Thus, $\omega$ is a real and positive value. $B(r)$ is the basis function in radial direction and it is given as

$$
B(r)=c_{1} J_{n}(\lambda r)+c_{2} Y_{n}(\lambda r)
$$

where $J_{\mathrm{n}}$ and $Y_{\mathrm{n}}$ are the Bessel functions of the first and second kind respectively. For a cylindrical combustor, $B(r)$ can be shown as

$$
B(r)=\sum_{m}^{\infty} J_{n}\left(\lambda_{n, m} r\right)
$$

Since $\frac{d J_{n}}{d r}\left(\lambda r_{o}\right)=0$ at $r=r_{o}$, i.e. the rigid wall boundary condition, $c_{2}=0 . \lambda_{n, m}$ is defined as the $(m+1)^{\text {th }}$ solution of $\frac{d J_{n}}{d r}\left(\lambda r_{o}\right)=0$.

For an annular combustor with $r_{i} \leq r \leq r_{o}, B(r)$ can be shown as

$$
\begin{aligned}
B(r) & =\sum_{m=1}^{\infty} \frac{d Y_{n}}{d r}\left(\lambda_{n, m} r_{o}\right) J_{n}\left(\lambda_{n, m} r\right) \\
& -\frac{d J_{n}}{d r}\left(\lambda_{n, m} r_{o}\right) Y_{n}\left(\lambda_{n, m} r\right)
\end{aligned}
$$

$\lambda_{n, m}$ is now the $(m+1)^{\text {th }}$ solution of

$$
\begin{gathered}
\frac{d Y_{n}}{d r}\left(\lambda_{n, m} r_{o}\right) \frac{d J_{n}}{d r}\left(\lambda_{n, m} r_{i}\right) \\
-\frac{d J_{n}}{d r}\left(\lambda_{n, m} r_{o}\right) \frac{d Y_{n}}{d r}\left(\lambda_{n, m} r_{i}\right)=0
\end{gathered}
$$

The angular frequencies as involved in Eq. $\omega=\omega_{l n m}$ are determined from

$$
k_{l n m}^{2}=\frac{\omega_{l n m}^{2}}{c_{S}^{2}}=k_{n m}^{2}+k_{z l}^{2}
$$

$k_{z l} L=l \pi$ is characterizing the longitudinal mode in an open-open or closed-closed combustor [43]. Here $l=1$, $2 \ldots$. Therefore, the acoustic frequency is intrinsically selected by the resonance nature of the combustor (i.e. depends on the combustor dimensions and geometric shapes). It acts like an 'amplifier' to combustion noise. Table 1 summarizes the theoretically predicted acoustic resonant frequencies of an annular shaped combustor as shown in Fig. 1(a). As revealed in Eq. (24), the acoustic resonant frequency depends strongly on the dimensions of the combustor and the speed of sound $c_{\mathrm{s}}$.

Table 1 Natural acoustic resonant frequencies of an annular combustor with an axial length of $110 \mathrm{~mm}$ and inner/outer diameters of $60 / 70 \mathrm{~mm}$ in the absence of chemical combustion. The speed of sound is set to $600 \mathrm{~m} / \mathrm{s}$.

\begin{tabular}{clll}
\hline$w_{\text {theo }} / 2 \pi(\mathrm{Hz})$ & $l$ & $\mathrm{~m}$ & $\mathrm{n}$ \\
\hline 1363.6 & 1 & 0 & 0 \\
1203.4 & 0 & 1 & 1 \\
2406.7 & 0 & 1 & 2 \\
3557.4 & 2 & 0 & 1 \\
\hline
\end{tabular}


Fig. 1(b) shows the mode-shapes of a cylindrical can-type combustor by plotting $\Phi(r, \theta)$ normalized with its maximum value. The transverse modes are spatially distributed along the cross-section of the combustor with $m$ and $n$ set to 3 . As longitudinal modes are concerned, open boundary condition corresponds to a pressure node [44], while closed-boundary is a velocity node. Thus the longitudinal mode is quite easy to understand by analogy analysis of a string (for example both ends fixed) vibration. It is not necessary to show the longitudinal mode-shapes in Fig. 1. However, our experimental measurement later will illustrate the longitudinal mode shape at the acoustic resonant frequency of the combustor.

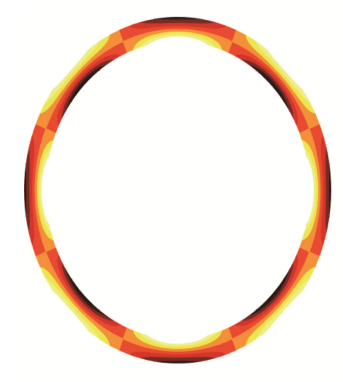

(a) $n=4, m=1$

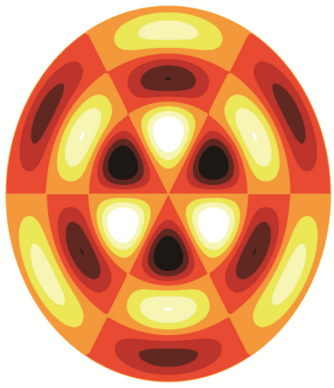

(b) $n=3, m=3$
Fig. 1 transverse acoustic wave mode-shapes in annular-type/ configuration (a) and can-type combustor (b)

\subsection{Intrinsic Unstable Nature}

Substituting Eq. (19) into Eq. (18) and separating the real and imaginary parts leads to

$$
\begin{aligned}
& c_{S}^{2}\left(\frac{\alpha \gamma-\delta Q_{T}}{\gamma}\right) \nabla^{2} F(r, \theta, z) \\
+ & {\left[3 \alpha \omega^{2}+\delta Q_{T} \alpha^{2}-\alpha^{3}-\delta Q_{T} \omega^{2}-\zeta\left(\alpha^{2}-\omega^{2}\right)\right] } \\
& F(r, \theta, z)=0 \\
& c_{S}^{2} \nabla^{2} F(r, \theta, z) \\
+ & {\left[\omega^{2}+2 \alpha \delta Q_{T}-3 \alpha^{2}-2 \zeta \alpha\right] F(r, \theta, z)=0 }
\end{aligned}
$$

If we define

$$
\chi \equiv \delta Q_{T} / \omega \text { and } G=\alpha / \omega \text { and } D=\zeta / \omega
$$

then eliminating $c_{s}^{2} \nabla^{2} F(r, \theta, z)$ between Eqs. (25a) and (25b) and applying Eq. (26) gives

$$
\begin{aligned}
2 G^{3} & +2 G+\chi\left(\frac{(1-\gamma)}{\gamma}\right)+\frac{2 G \chi^{2}}{\gamma} \\
& -\chi G^{2}\left(1+\frac{3}{\gamma}\right)+D\left[G^{2}-\frac{2 G \chi}{\gamma}+1\right]=0
\end{aligned}
$$

Eq. (27) is the characteristic governing relationship between thermal energy input $\chi \equiv \delta Q_{T} / \omega$ and the acoustic disturbance evolution $G=\alpha / \omega$ for a given $\gamma, \omega$ and $D$.
The extreme values of $G$ (i.e. $G_{\text {ex }}$ ) can be determined by differentiating Eq. (27) with $\chi$ by setting $\partial G / \partial \chi=0$ as

$$
\begin{array}{r}
4 G_{e x} \chi_{e x} / \gamma+\frac{(1-\gamma)}{\gamma}-G_{e x}^{2}\left(\frac{\gamma+3}{\gamma}\right)-D \frac{2 G_{e x}}{\gamma}=0 \\
\chi_{\max }=\frac{G_{\max }^{2}(\gamma+3)-(1-\gamma)}{4 G_{\max }} \\
G_{\max }=\frac{\frac{4 \chi_{\max }}{\gamma} \pm \sqrt{\left(\left(\frac{4 \chi_{\max }}{\gamma}\right)^{2}+\frac{4(1-\gamma)(\gamma+3)}{\gamma^{2}}\right)}}{2\left(1+\frac{3}{\gamma}\right)}
\end{array}
$$

When the acoustic damping/losses $D$ is assumed to be negligible, i.e. $D=0$, the maximum growth rate $G_{\mathrm{ex}}$ and the corresponding $\chi_{e x}$ can be determined by using Eqs. (27) and (28).

It is expected that the experimentally measured $G_{\exp }$ in any practical combustion system is smaller than the theoretically predicted $G_{\max }$, which is derived by assuming the losses $D=0$. The difference between $G_{\max }$ and $G_{\exp }$ is due to the acoustic damping/losses $D$ involved in the inlet and outlet boundaries and/or viscous boundary layers. This finding will be validate by comparing the present estimation of $G_{\max }$ with the experimental date available in the literature in Sect. 3. The acoustic losses coefficient $D$ is varied with the change of operating conditions of the combustion system. Thus it is quite challenging to experimentally determined.

\subsection{Acoustical Energy Growth Rate}

Acoustical Energy $W_{\text {a }}$ consist of both kinetic energy and potential energy [44]. It is given as

$$
\begin{aligned}
W_{a}(t) & =\frac{1}{2} \bar{\rho} \int_{z=0}^{z=L} \mathrm{~d} z \int_{0}^{2 \pi} \mathrm{d} \theta \int_{r=r_{i}}^{r=r_{o}}(\nabla \phi)^{2} r \mathrm{~d} r \\
& +\frac{1}{2} \frac{\bar{\rho}}{c_{S}^{2}} \int_{z=0}^{z=L} \mathrm{~d} z \int_{0}^{2 \pi} \mathrm{d} \theta \int_{r=r_{i}}^{r=o}(\partial \phi / \partial t)^{2} r \mathrm{~d} r
\end{aligned}
$$

The integral of Eq. (29) could be determined by using Green's theorem

$$
\iiint(\nabla \phi)^{2} \mathrm{~d}^{3} r+\iiint \phi \nabla^{2} \phi \mathrm{d}^{3} r=\iint(\phi \nabla \phi)_{\text {norm }} \mathrm{d}^{2} r
$$

when only transverse acoustic mode is considered, $\iiint(\Phi \nabla \Phi)_{n o r m} d^{2} r=0$, since the values of $(\Phi \nabla \Phi)_{\text {norm }}$ is zero over the surfaces and it is also zero over the combustor inner surface wall. When acoustically open-open/closed-closed combustor is considered, $\iiint(\Phi \nabla \Phi)_{\text {norm }} d^{2} r \mid \begin{aligned} & z=L \\ & z=0\end{aligned}=0$. 
Thus Eq. (29) can be further simplified into

$$
\begin{aligned}
W_{a}(t)= & \frac{1}{2} \frac{\bar{\rho}}{c_{S}^{2}} \int_{z=0}^{z=L} \mathrm{~d} z \int_{0}^{2 \pi} \mathrm{d} \theta \\
& \int_{r=r_{i}}^{r=r_{o}}\left\{\left(\frac{\partial \phi}{\partial t}\right)^{2}-\phi \frac{\partial^{2} \phi}{\partial t^{2}}\right\} r \mathrm{~d} r
\end{aligned}
$$

Finally, the acoustical energy $W_{a}(t)$ can be shown as

$$
\begin{aligned}
W_{a}(t)= & \pi \bar{\rho} \phi_{0}^{2} e^{2 \alpha t} \Re \\
& \left\{\int_{z=0}^{z=L} e^{j z k_{z l}} \mathrm{~d} z \int_{r=r_{i}}^{r=o} B^{2}\left(\frac{\omega r}{c_{S}}\right) \frac{\omega r}{c_{S}} \mathrm{~d}\left(\frac{\omega r}{c_{S}}\right)\right\} \\
= & W_{a 0} e^{2 \alpha t} \geq 0
\end{aligned}
$$

where $W_{a 0}=W_{a}(t=0)$. The acoustical energy growth rate is given as

$$
\frac{1}{W_{a}(t)} \frac{\mathrm{d}\left(W_{a}(t)\right)}{\mathrm{d} t}=2 \alpha
$$

Here $\alpha$ is the growth rate of the acoustic disturbances. When $\alpha$ is negative, it describes the decaying process of acoustic disturbances. Eq. (33) reveals that the total energy of an acoustic wave in the combustion system varies with time as $e^{2 \alpha t}$. In practice, such acoustical energy [45] can be amplified and extracted to produce mechanical work or for cooling purpose [46, 47], which are also known as thermos-acoustic Stirling engine [46] or fridge [47].

\section{Results and Discussion}

The mathematical solutions/roots to Eq. (27) are illustrated in Fig. 2 in the absence of the acoustic dissipation/damping, i.e. $D=0$, as $\gamma$ is set to 3 different values. It can be seen that there are a pair of complex conjugate roots for any given $\gamma$ as denoted by the green and red dots. The roots denoted by the blue dots are real values with the imaginary parts being zero. It reveals that there is a physical solution to the combustion-driven noise equation. In physics that the combustion noise could be present over a broad band frequency range. Note that $\gamma=2.4$ and $\gamma=3.4$ are not practical, since the specific heat ratio $\gamma$ is typically in the range of 1.2-1.4. Here we conduct example calculations by setting $\gamma=2.4$ and 3.4.

In order to gain insights on the nature of the combustion noise, the growth rate $G$ of combustion-driven noise is determined for the real roots of Eq. (27). This is illustrated in Fig. 3. It is apparent that as the imaginary part $\mathcal{X}(G)$ is 0 , then $G=\mathfrak{R}(G)+\mathrm{j} \mathcal{X}(G)=\mathfrak{R}(G)$. Furthermore, $\mathscr{R}(G)$ is greater than 0 , no matter what value $\gamma$ is set to. This reveals that the combustor is intrinsically/naturally unstable, when there is no acoustic damping/dissipation, i.e. $D=0$. $G$ is found to be increased first and then decreased. There is a maximum growth rate $G_{\max }=(\alpha / \omega)_{\max }$ for each given $\gamma$. At $\gamma=1.4$ (corresponding to the general air properties at ambient temperature and pressure), $G_{\max }=9.2 \times 10^{-2} \mathrm{rad} / \mathrm{s}^{2}$. This is the maximum possible growth rate of any combustion-driven acoustic disturbances. In practice, the experimentally measured maximum growth rate with a unit of $\mathrm{rad} / \mathrm{s}^{2}$ should be less than $G_{\max }=9.2 \times 10^{-2}$. The difference between the theoretically predicted maximum growth rate and the experimental measured one $G_{\text {exp }} \equiv$ $(\alpha / \omega)_{\exp }$ is resulting from the acoustic damping/losses are present in any practical combustor losses involving with the acoustic wave propagation and the boundaries (acoustic impedance changes).

Table 2 summarizes and compares the present predicted $G_{\max }$ with the experimental data available in the literature. It can be seen from Table 2 that the measured $G_{\text {exp }} \equiv(\alpha / \omega)_{\exp }$ is less than the theoretical $G_{\text {theo }} \equiv(\alpha / \omega)_{\text {theo }}$, as a number of types of hydrocarbon fuels are supplied. Note that the fossil fuels used in the lab-scale combustors are the typical ones applied in energy and propulsion

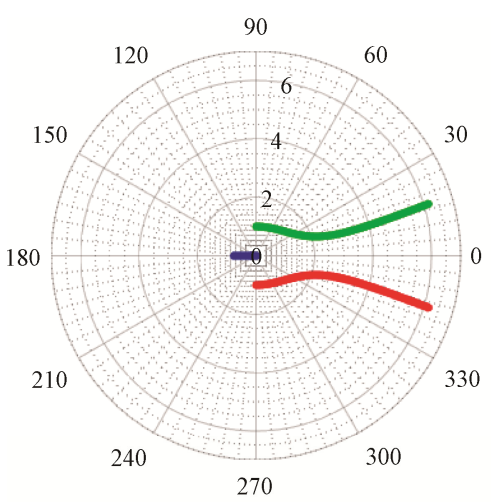

(a) $\gamma=1.40$

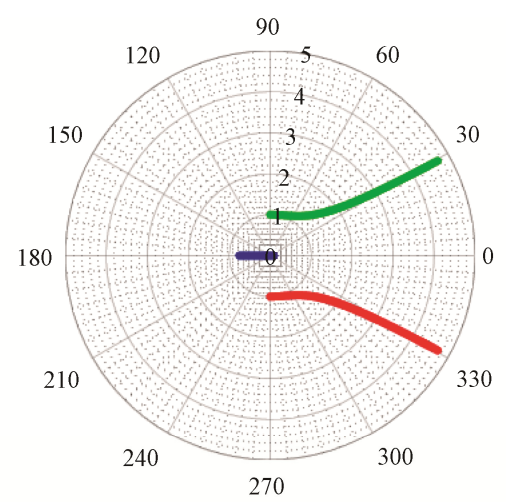

(b) $\gamma=2.40$

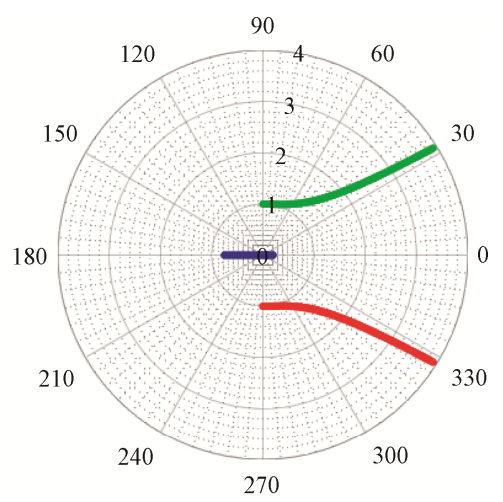

(c) $\gamma=3.40$

Fig. 2 Mathematical solutions of the $3^{\text {rd }}$-order polynomial combustion-driven noise Eq. (27), as $\chi$ is varied from 0 to 6 , the acoustic losses/dissipation $D$ is set to $D=0$, and $\gamma$ is set to 3 different values 
industries. Further validation is conducted on a well-design T-shaped combustor with a cooling perforated pipe implemented as described in Sect. 4.

Fig. 4 shows the variation of the extreme $G_{\text {ex }}$ with the corresponding $\chi_{\mathrm{ex}}$, as described in Eq. (28a). It can be seen that there are two roots of $G_{\text {ex }}$ as shown in Fig. 4(a) and (b). In either case, the extreme $G_{\text {ex }}$ could be complex values, as $\chi_{\text {ex }}$ is below a critical value. However, furthering increasing $\chi_{\mathrm{ex}}$, above the critical value leads to

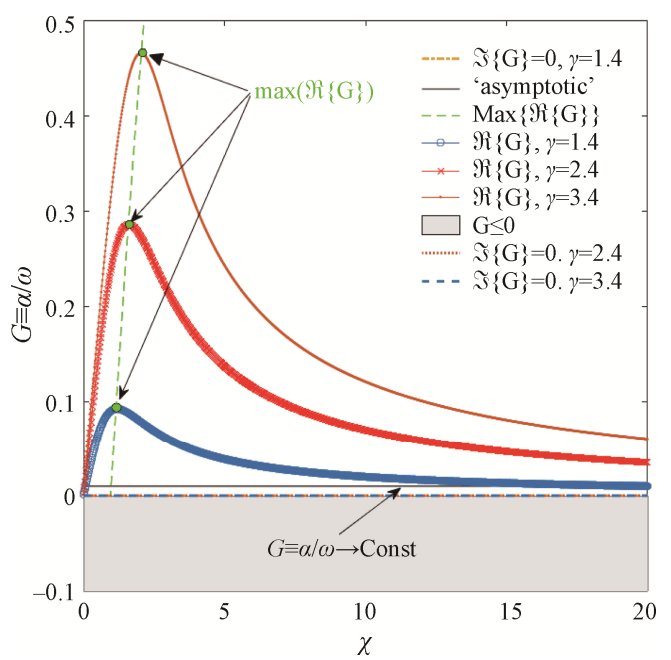

Fig. 3 Variation of the normalized growth rate $G$ (real roots only) with $\chi$, as the acoustic dissipation $D$ is set to $D=0$, and $\gamma$ is set to 3 different values, i.e. $\gamma=1.4, \gamma=2.4$ and $\gamma=3.4$. Here, $\gamma=2.4$ and $\gamma=3.4$ are used for demonstration calculations. the imaginary part of $G_{\mathrm{ex}}$ is zero. The real part is positive. One roots is increased with $\chi_{\mathrm{ex}}$, and the other root is decreased. Fig. 4(c) shows the variation of $\chi_{\mathrm{ex}}$ with $G_{\mathrm{ex}}$. It is obvious that there is a minimum $\chi_{\mathrm{ex}}$.

Fig. 5 shows the effect of acoustic damping/losses on the combustion-driven acoustics growth rate $G$. It can be seen from Fig. 5(a) and (b) that there are a pair of complex conjugate roots of Eq. (27). Real roots are present as shown in Fig. 5(c), since the corresponding imaginary parts are all zeros. It can be seen that below the critical value of $\chi_{\mathrm{cr}}$, as denoted by the circle solid markers in different colours, the growth rate $G$ is below 0 . It means that the acoustic disturbances are decaying exponentially. When the heat capacity $\chi$ is increased above $\chi_{\mathrm{cr}}$, the growth rate is positive and increased with $\chi$. The acoustic disturbances in the system are growing exponentially. Finally, increasing specific heat ratio $\gamma$ leads to a decrease of $\chi_{\mathrm{cr}}$. In physics, it means that the combustion system is more prone to be unstable

Fig. 6 illustrates the time evolution of the acoustical energy and the acoustic disturbances, as $\omega=200, \gamma=1.4$ and $G$ is set to 3 different values. It can be seen that the acoustical energy $W_{\mathrm{a}}(\mathrm{t})$ is either growing or decaying exponentially, depending on the growth rate $G$. Furthermore, the growth rate is $100 \%$ faster than that of the acoustic disturbances. For any practical combustion system, a stable combustion is desirable. In other words, the system with the acoustical energy features as illustrated in Fig. 6(c) is more attractive, since the acoustic disturbances in such system are quickly decaying with time.

Table 2 summary of experimentally measured growth rate $G_{\exp } \equiv(\alpha / \omega)_{\exp }$ and the theoretical predicted maximum $G_{\max } \equiv(\alpha / \omega)_{\max }$.

\begin{tabular}{|c|c|c|c|c|c|}
\hline Authors \& Refs. & $\begin{array}{l}\text { Fuel/chemical } \\
\text { compounds }\end{array}$ & $\begin{array}{l}\text { Measured } \\
\text { Dominant } \\
\omega / 2 \pi(\mathrm{Hz}) \\
\end{array}$ & $\begin{array}{l}\text { Measured } \\
\text { Growth } \\
\text { rate } \alpha(1 / \mathrm{s})\end{array}$ & $\begin{array}{l}\text { Experimentally } \\
\text { Measured }(\alpha / \omega)_{\exp } \\
\left(\mathrm{rad} / \mathrm{s}^{2)}\right.\end{array}$ & $\begin{array}{c}\text { Theoretically } \\
\text { estimated }(\alpha / \omega)_{\max } \\
\left(\mathrm{rad} / \mathrm{s}^{2}\right)\end{array}$ \\
\hline Noiray et al. [25] & $\mathrm{CH}_{4}$ & 150 & 8.1 & $8.6 \times 10^{-3}$ & $9.2 \times 10^{-2}$ \\
\hline Mejia et al. [22] & $\mathrm{CH}_{4}$ & 56 & $1.8 \sim 4.9$ & $5.1 \sim 13.9 \times 10^{-3}$ & $9.2 \times 10^{-2}$ \\
\hline Morgans and Dowling [32] & $\mathrm{C}_{2} \mathrm{H}_{4}$ & 200 & 8.7 & $6.9 \times 10^{-3}$ & $9.2 \times 10^{-2}$ \\
\hline Blonbou et al. [33] & $\mathrm{C}_{3} \mathrm{H}_{8}$ & 235 & 13.3 & $9.0 \times 10^{-3}$ & $9.2 \times 10^{-2}$ \\
\hline Bloxsidge, et al. [34] & $\mathrm{C}_{2} \mathrm{H}_{4}$ & 77 & 33 & $6.8 \times 10^{-2}$ & $9.2 \times 10^{-2}$ \\
\hline Heckl [35] & Electrical Heater & 170 & 5.96 & $5.6 \times 10^{-3}$ & $9.2 \times 10^{-2}$ \\
\hline Yu et al. [36] & JP8 Jet fuel & 1300 & 134 & $1.64 \times 10^{-2}$ & $9.2 \times 10^{-2}$ \\
\hline Gulati and Mani [37] & $\mathrm{CH}_{4}$ & 262 & 1.23 & $0.74 \times 10^{-3}$ & $9.2 \times 10^{-2}$ \\
\hline Burnley and Culick [38] & Unknown & 904 & 25 & $4.4 \times 10^{-3}$ & $9.2 \times 10^{-2}$ \\
\hline Eckstein and Sattelmayer [39] & Ethanol $\left(\mathrm{C}_{2} \mathrm{H}_{5} \mathrm{OH}\right)$ & 129 & 40 & $4.9 \times 10^{-2}$ & $9.2 \times 10^{-2}$ \\
\hline Motheau, et al. [40] & $\begin{array}{c}\text { Kerosene } \\
\mathrm{C}_{12} \mathrm{H}_{26}\end{array}$ & 429 & 12 & $4.5 \times 10^{-3}$ & $9.2 \times 10^{-2}$ \\
\hline Kim and Hochgreb [26] & $\mathrm{CH}_{4}$ & 186 & 10.2 & $8.7 \times 10^{-3}$ & $9.2 \times 10^{-2}$ \\
\hline Zhao et al. [41] & $\mathrm{C}_{3} \mathrm{H}_{8}$ & 245 & 6.1 & $3.9 \times 10^{-3}$ & $9.2 \times 10^{-2}$ \\
\hline
\end{tabular}




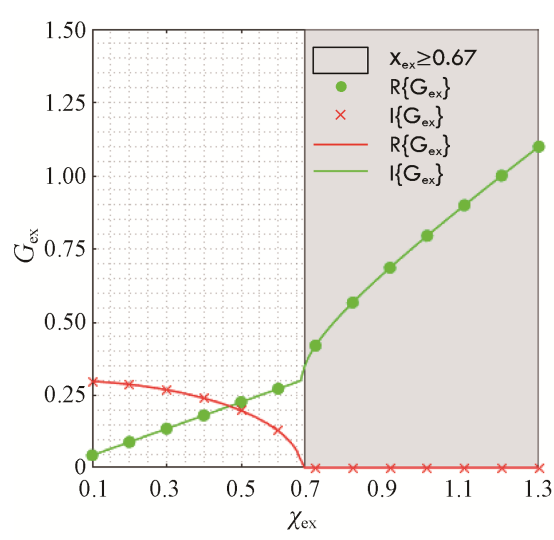

(a) $1^{\text {st }}$ root of $G_{\text {ex }}$

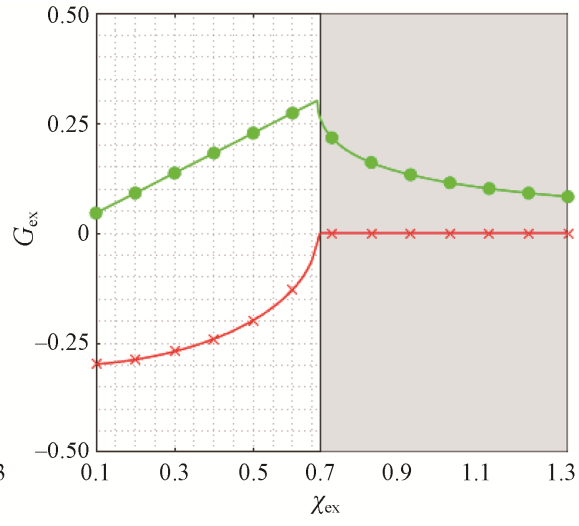

(b) $2^{\text {nd }}$ root of $G_{\text {ex }}$

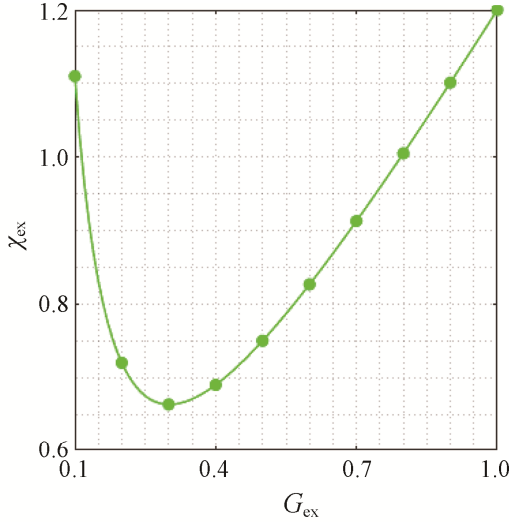

(c) $\chi_{\mathrm{ex}}$ varied with $G_{\mathrm{ex}}$

Fig. 4 Variation of the extreme value of $G_{\text {ex }}$ with the corresponding $\chi_{\text {ex }}$ as described in Eq. (28a), as the acoustic dissipation $D$ is set to $D=0$, and $\gamma$ is set 1.4 i.e. $\gamma=1.4$.
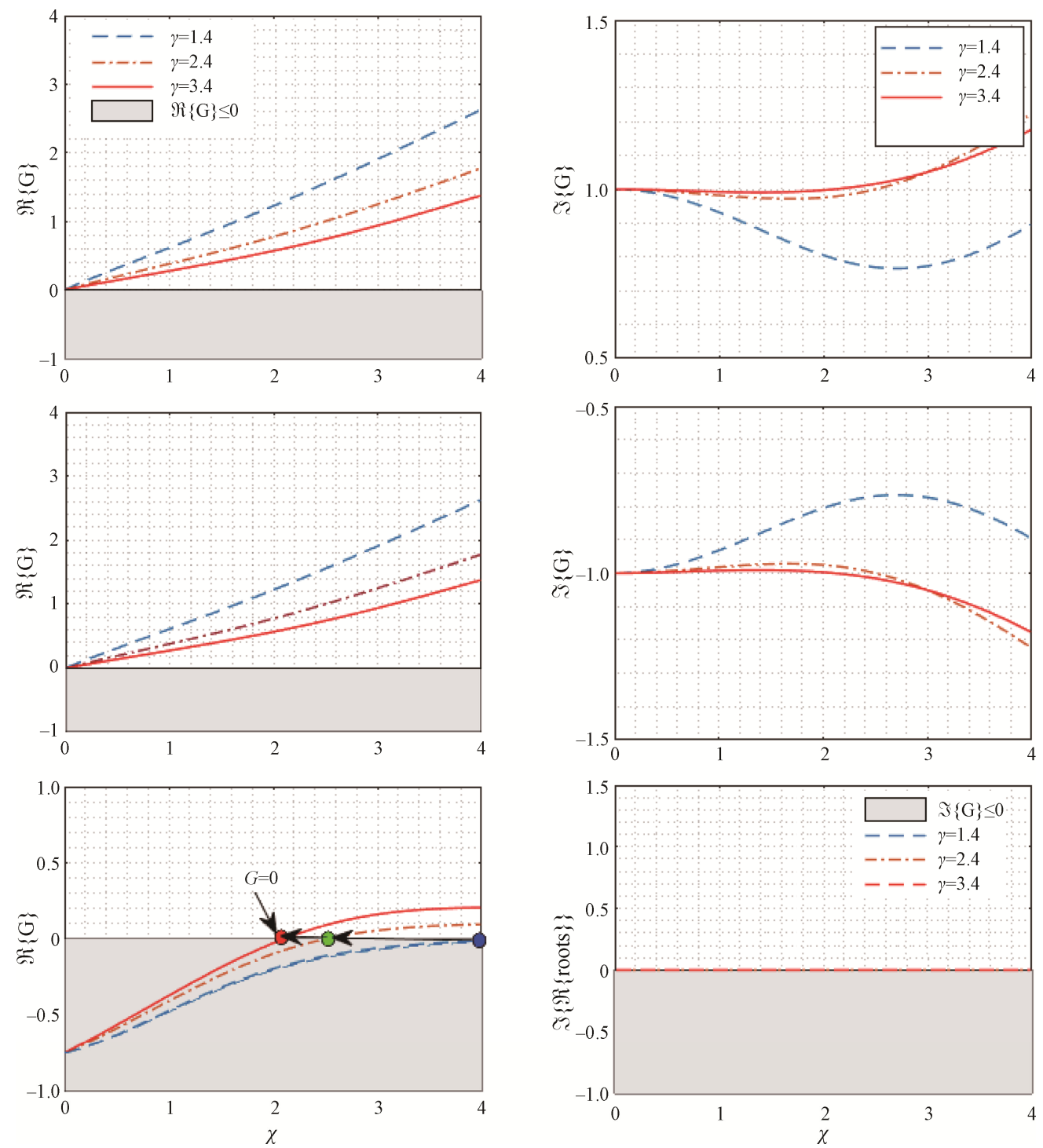

Fig. 5 Mathematical roots of the $3^{\text {rd }}$-order polynomial combustion-driven noise Eq. (27), as the acoustic dissipation $D$ is set to $D=1$, and $\gamma$ is set to 3 different values. (a) and (d) the real and imaginary parts of the $1^{\text {st }}$ root of Eq. (27), (b) and (e) the real and imaginary parts of the $2^{\text {nd }}$ root, (c) and (f) the real and imaginary parts of the $3^{\text {rd }}$ root 


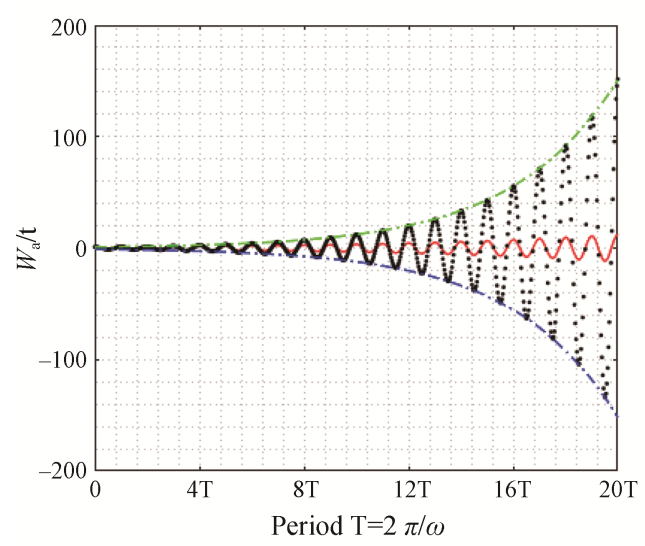

(a) $\omega=200, G=0.01$

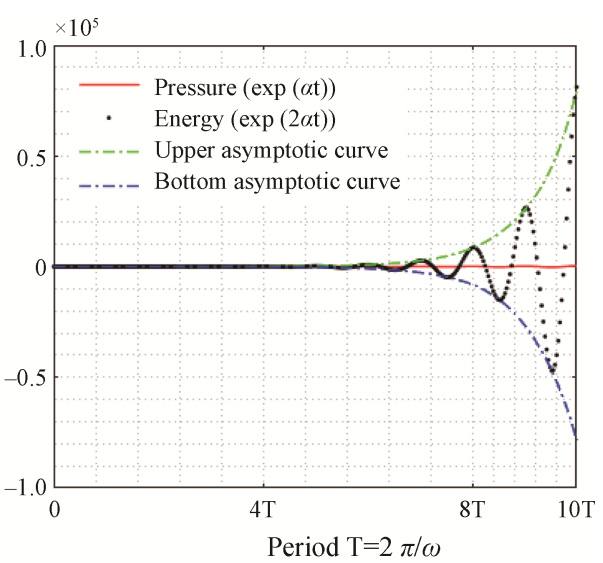

(b) $\omega=200, G=0.09$

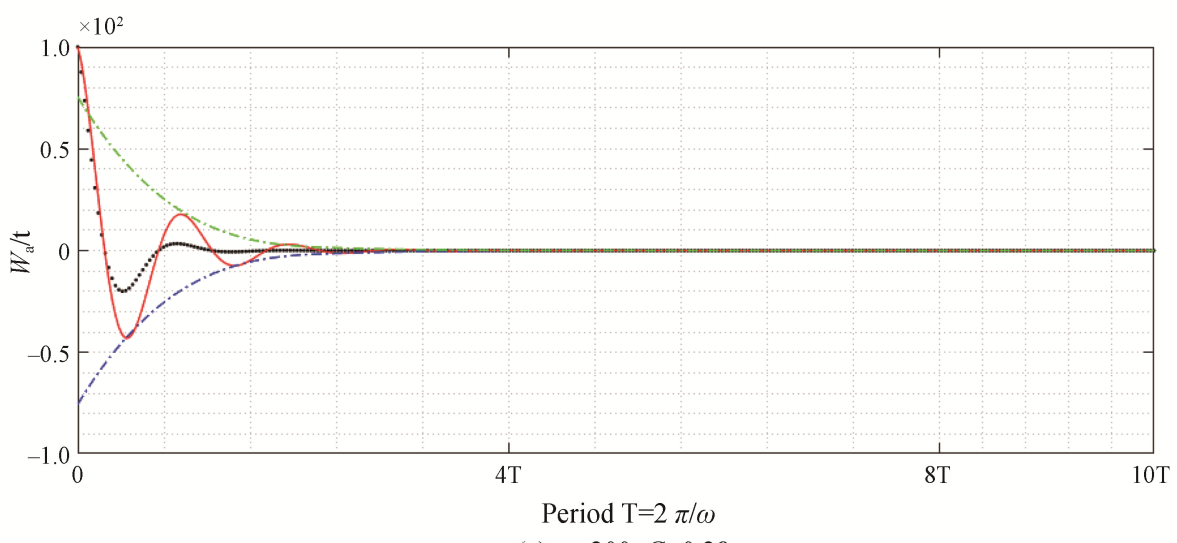

(c) $\omega=200, G=0.28$

Fig. 6 Time evolution of the acoustic disturbances and acoustical energy $W_{\mathrm{a}}$, as $\omega=200, \gamma=1.4$ and $G$ is set to 3 different values

\section{Experimental Studies}

Experimental studies are conducted on a well-designed T-shaped combustion system to measure the growth rate $G \equiv \alpha / \omega$ of combustion-driven oscillations normalized by its frequency. This test rig is a spin-off of classical Rijke-type combustor [42, 43]. The schematic of the experimental setup is shown in Fig. 7. The bottom stem of the T-combustor is $30 \mathrm{~cm}$ long and has an inner diameter of $50 \mathrm{~mm}$. The bifurcating branches sharing the same inner diameter of $45 \mathrm{~mm}$ and the same axial length of $50 \mathrm{~cm}$. A perforated pipe made of acrylic materials is attached to the T-shaped combustor. To protect the perforated pipe with an axial length of $15 \mathrm{~cm}$ and inner diameter of $45 \mathrm{~mm}$, a centrifugal pump is implemented. It pumps ambient air through the perforated pipe joining the combustion flow and flowing out through the right-hand-side horizontal branch of the T-combustor. The cooling flow rate is in the range of $0 \mathrm{~m}^{3} / \mathrm{s}$ to 0.1074 $\mathrm{m}^{3} / \mathrm{s}$. The perforated pipe involves with 112 circle-shaped orifices with a diameter of $3 \mathrm{~mm}$. these orifices are distributed equally in space along the pipe surface. Acoustic pressure measurements are conducted by using 2 arrays of B\& K 4957 microphones with a sensitivity of
$11.2 \mathrm{mV} / \mathrm{Pa}$. The microphones are calibrated with B\&K Pistonphone (124dB @250 Hz, Type 4228). A premixed conical-shaped flame is confined at $13.5 \mathrm{~cm}$ away from the bottom open inlet.

The measured combustion-driven oscillations from the 4 pressure sensors are shown in Fig. 8. It shows the time evolution of the phase diagram/plot between the pressure $p^{\prime}(\mathrm{t})(\mathrm{Pa})$ and its gradient $\mathrm{dp}(\mathrm{t}) / \mathrm{dt}(\mathrm{Pa} / \mathrm{s})$. It can be seen that the combustion-driven oscillations are limit cycles initially at $t \leq 20 \mathrm{~s}$, as revealed by the circle-shaped phase plot. This is known as Rijke-type thermoacoustic phenomena as discussed by Feldman JR [43]. However, as the cooling flow rate is increased to $5.5 \mathrm{~m} / \mathrm{s}$, the oscillations are attenuated (see $30 \mathrm{~s} \leq t \leq 60 \mathrm{~s}$ ). At $t=60 \mathrm{~s}$, the cooling flow rate is suddenly reduced to $0 \mathrm{~m} / \mathrm{s}$. The combustion-driven oscillations quickly grow into limit cycles.

Time evolution of the local peaks/amplitude of the experimentally measured acoustic pressure fluctuations [42, 43] is shown in Fig. 9. Fig. 9(a) and (b) illustrates the two sensors measurements on left- and right-hand-side bifurcating branches respectively. The growth rate $\alpha$ is determined by fitting the linear equation (denoted by the dash line). The frequency of the 
measured acoustic fluctuations is shown in Fig. 9(c) in the pressure spectrum. It is approximately $215 \mathrm{~Hz}$. There are harmonic peaks in the spectrum. However, these peaks magnitudes are at least 1 order lower than the dominant peak at $215 \mathrm{~Hz}$. With the dominant oscillation frequency determined, the mode-shape as experimentally measured is shown in Fig. 9(d). It can be seen that the combustion system is a standing-wave one [44, 45]. This

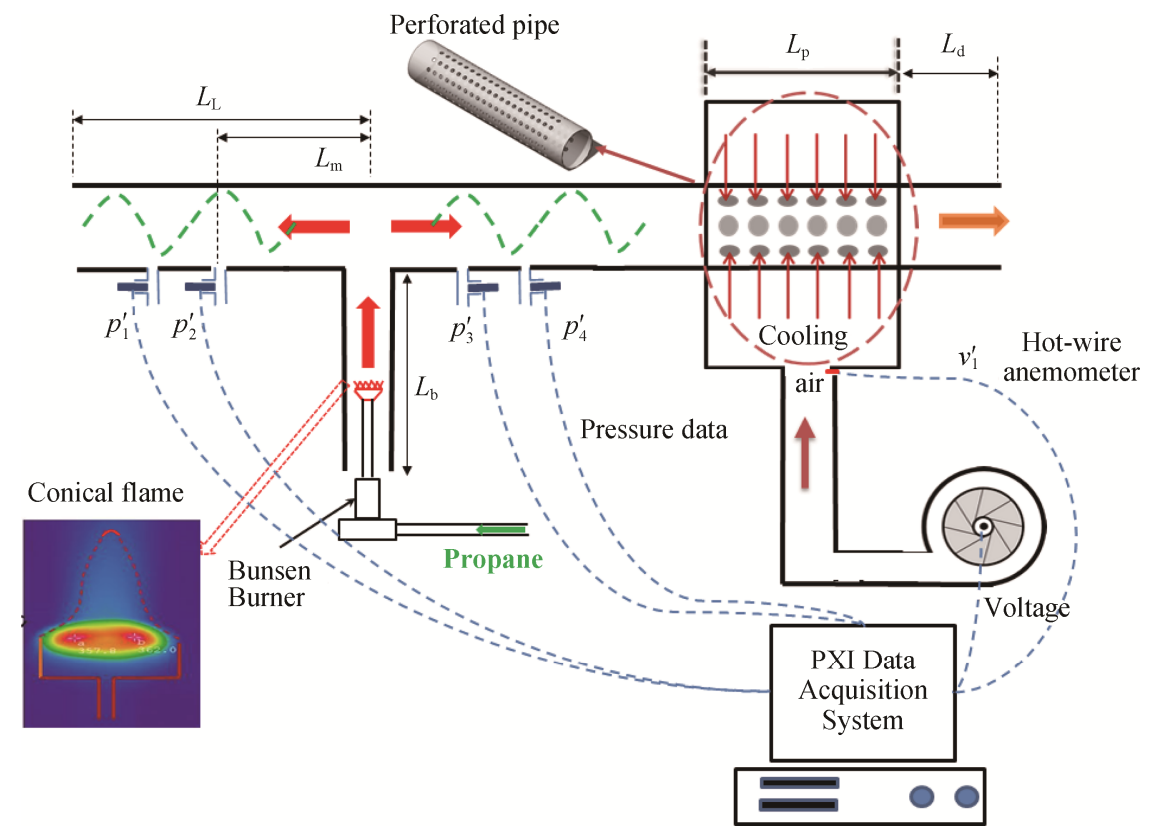

Fig. 7 Schematics of the experimental rig of T-shaped combustion system with a cooling perforated pipe applied
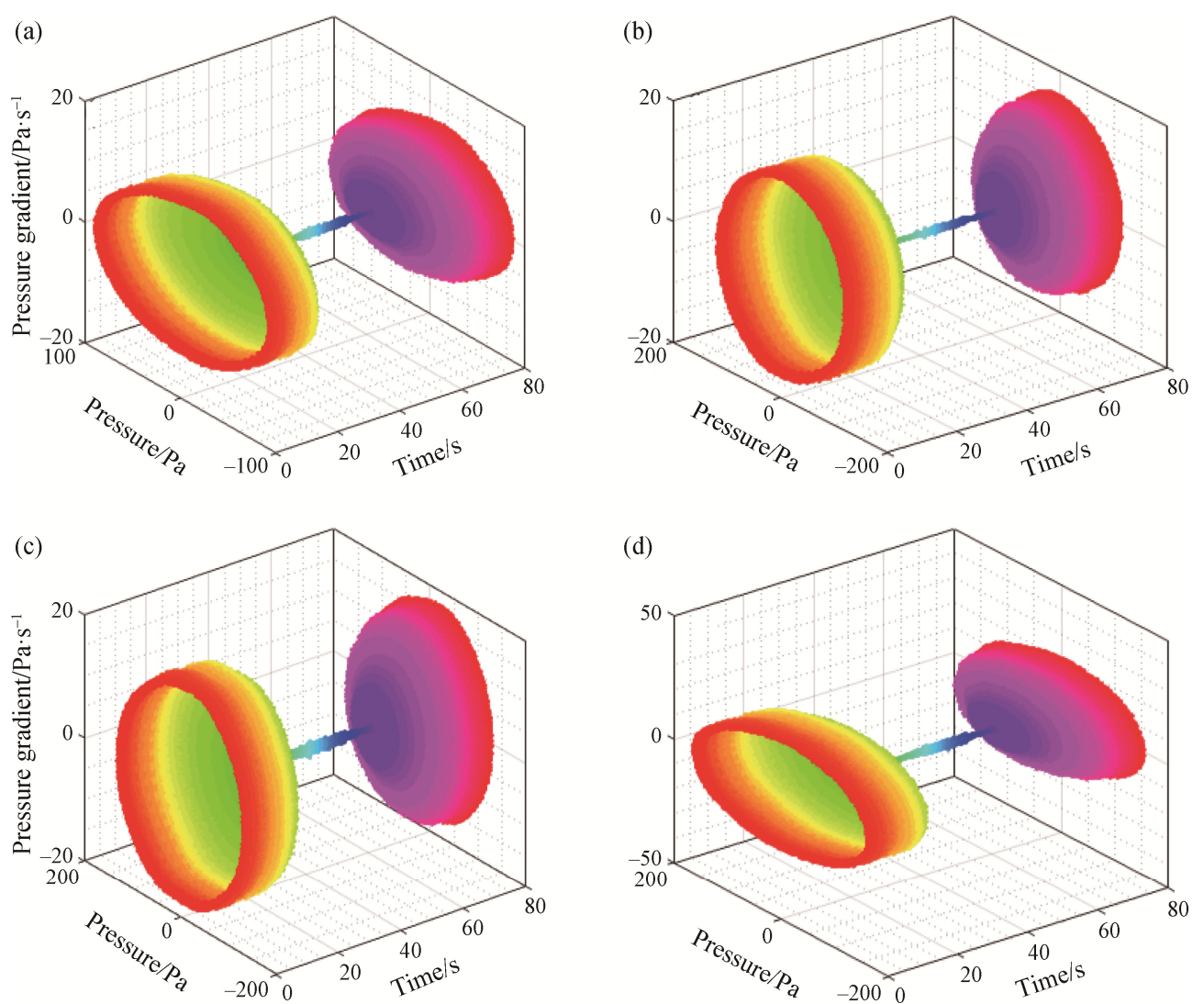

Fig. 8 Experimentally measured combustion-driven pulsating oscillations from sensor 1 (a) and from sensor 2 (b), sensor 3 (c) and sensor 4 (d) 
Dan Zhao et al. Thermodynamics-Acoustics Coupling Studies on Self-excited Combustion Oscillations Maximum Growth Rate 11

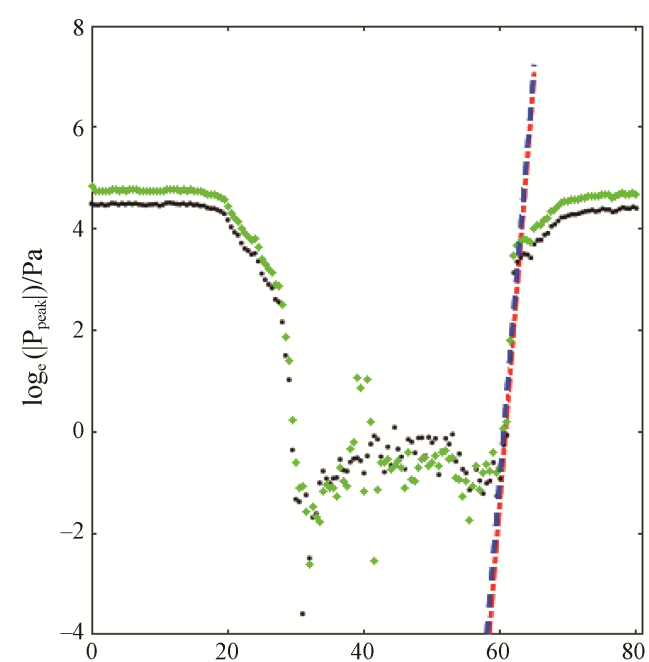

(a) $1.24 \times 10^{-3}$

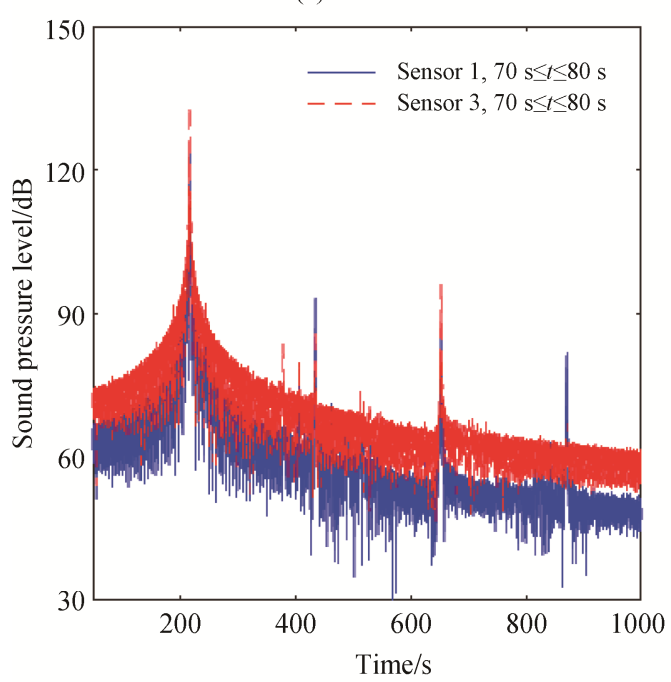

(c) $1.23 \times 10^{-3}$

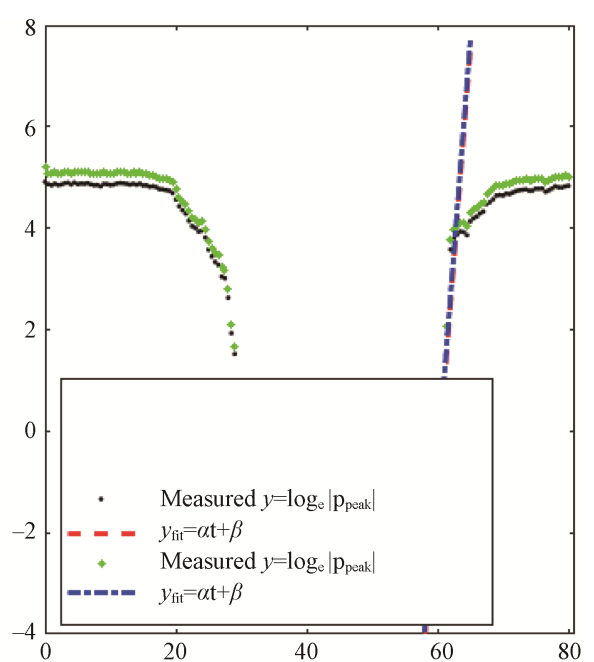

(b) $1.18 \times 10^{-3}$

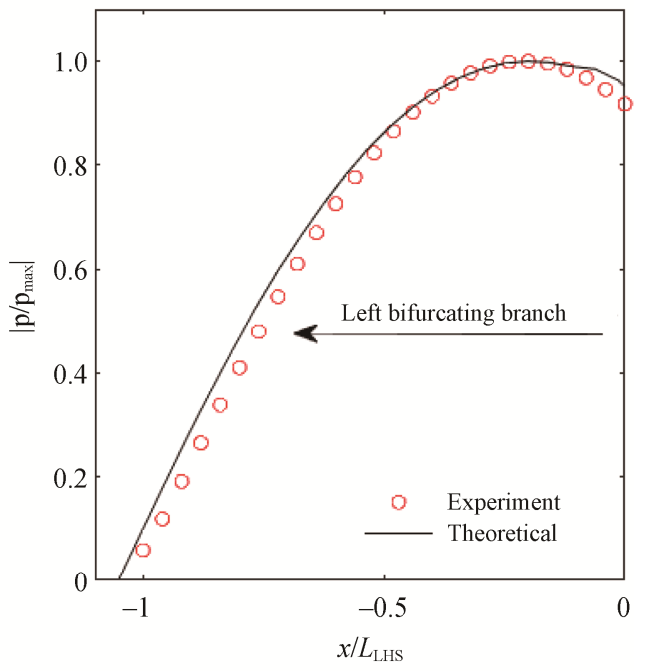

(d) $\times 10^{-3}$

Fig. 9 Experimentally measured growth rate $\alpha$ of the dominant pulsating oscillation from sensor 1: $\alpha=1.676$ and $\beta=-100.16$ (a); sensor $2 \alpha=1.603$ and $\beta=95.38$ (b), sensor 3: $\alpha=1.668$ and $\beta=99.24$ (c) and sensor $4: \alpha=1.669$ and $\beta=-99.09$ (d). The measured dominant frequency is $215 \mathrm{~Hz}$. The normalized growth rate $G_{\exp } \equiv(\alpha / \omega)_{\exp }$

confirms that the acoustic frequency is intrinsically selected according to the longitudinal resonance nature of the combustor. In practice, such self-sustained limit cycle oscillations [46-49] are desirable in thermoacoustic Stirling engines [46] and refridges [47].

\section{Conclusions}

We have shown how hydrocarbon-fuelled flame can generate self-excited combustion oscillations, and the oscillations frequency selection due to the acoustic resonance nature of the combustor. The physics behind the chemical reaction-thermodynamics-acoustics [50] is theoretically examined and experimentally validated. It is found that any combustor is intrinsically unstable in the absence of acoustic losses/dissipation (i.e. $D=0$ ). In physics, it means that small-amplitude acoustic disturbances at any frequency $\omega$ will be exponentially amplified. Furthermore, there is a maximum growth rate $\alpha_{\max }$ of the combustion-driven acoustic disturbances. It is estimated to be $G_{\max } \equiv \max \{\alpha / \omega\}=9.2 \times 10^{-2} \mathrm{rad} / \mathrm{s}^{2}$ at the specific heat ratio $\gamma=1.4$ and $D=0$. Comparing with the experimentally measured data available in the literature reveals that the theoretically predicted maximum growth rate is greater than the measured ones as expected. This is due to the presence of the acoustic damping/losses in practical combustion systems resulting from viscous boundary layer and the acoustic impedance change at the inlet and outlet boundaries (i.e. boundary losses).

The impact of the acoustic damping is examined by introducing an acoustic loss term. It is found that there is a critical heat capacity $\chi_{\mathrm{cr}} \equiv(\mathrm{d} Q / \mathrm{d} T)_{\mathrm{cr}}$. Below the critical 
value, the system is stable. Acoustic disturbances will be exponentially decaying with time. Finally, the combustion oscillations is naturally selected due to the acoustic resonance features of the combustor with specified dimensions. The growth rate $G_{\exp } \equiv(\alpha / \omega)_{\exp }$ and the acoustic resonant frequency $\omega$ are confirmed by our well-design T-shaped combustion system with a cooling-flow perforated pipe implemented. The present work sheds lights on the fundamental physics and mechanisms underlying the self-excited combustion oscillations [51-52] in any hydrocarbon-fuelled combustors. The theoretical maximum growth rate is applicable to a combustion system with other types of fuels, such as Hydrogen and Ammonia.

\section{Acknowledgements}

We gratefully acknowledge the financial support from the University of Canterbury, New Zealand (grant no. 452STUPDZ) and Singapore National Research Foundation (grant no. NRF2016 NRF-NSFC001-102), and National Natural Science Foundation of China (11661141020).

\section{References}

[1] Bragg, S.L., Noise and oscillations in jet engines. Nature, 1964, 201(491): 123-124.

[2] Rayleigh, L., The explanation of certain acoustical phenomena. Nature, 1878, 18: 319-321.

[3] Lieuwen, T.C., Unsteady combustor physics, Cambridge University Press, Cambridge, 2012.

[4] Park, S., Choi, G.M. and Tanahashi, M., Demonstration of a gas turbine combustion-tuning method and sensitivity analysis of the combustion-tuning parameters with regard to NOx emissions. Fuel, 2019, 239: 1134-1142.

[5] Hashimoto T, Shibuya H, Gotoda H, Ohmichi Y, Matsuyama S., Spatiotemporal dynamics and early detection of thermoacoustic combustion instability in a model rocket combustor. Physical Review E, 2019, 99(3): 032208.

[6] Sakaki K, Funahashi T, Nakaya S, Tsue M, Kanai R, Suzuki $\mathrm{K}$, Inagawa $\mathrm{T}$, Hiraiwa $\mathrm{T}$, Longitudinal combustion instability of a pintle injector for a liquid rocket engine combustor. Combustion and Flame, 2018, 194: 115-127.

[7] Xiong J, Morgan H, Krieg J, Liu F, Sirignano WA, Nonlinear Combustion Instability in a Multi-injector Rocket Engine. AIAA Journal, 2020, 58(1): 219-35.

[8] Guan Y, Gupta V, Wan M, Li LK, Forced synchronization of quasiperiodic oscillations in a thermoacoustic system. Journal of Fluid Mechanics, 2019, 879: 390-421.

[9] Moon K, Jegal H, Gu J, Kim KT, Combustion-acoustic interactions through cross-talk area between adjacent model gas turbine combustors. Combustion and Flame, 2019, 202: 405-416.

[10] Magri L, Juniper MP, Moeck JP, Sensitivity of the Rayleigh criterion in thermoacoustics. Journal of Fluid Mechanics, 2020, 882.

[11] Johnson M, Agrawal AK, Effects of Porous versus Solid Inserts Pertaining to Instability Mitigation in Lean Direct Injection Combustion. AIAA SciTech 2019 Forum, 2019, p. 0992.

[12] Nagarajan B, Baraiya NA, Chakravarthy SR, Effect of inlet flow turbulence on the combustion instability in a premixed backward-facing step combustor. Proceedings of the Combustion Institute, 2019, 37(4): 5189-5196.

[13] Sogaro FM, Schmid PJ, Morgans AS, Thermoacoustic interplay between intrinsic thermoacoustic and acoustic modes: non-normality and high sensitivities. Journal of Fluid Mechanics, 2019, 878: 190-220.

[14] Schulze M, Sattelmayer T, Eigenvalue Analysis for the Prediction of Initial Growth Rates of Thermoacoustic Instability in Rocket Motors. 53rd AIAA Aerospace Sciences Meeting, 2015, pp. 1606.

[15] Nagaraja S, Kedia K, Sujith RI, Characterizing energy growth during combustion instabilities: Singularvalues or eigenvalues? Proceedings of the Combustion Institute, 2009, 32(2): 2933-2940.

[16] Schulze M, Sattelmayer T, Linear stability assessment of a cryogenic rocket engine. International Journal of Spray and Combustion Dynamics, 2017, 9(4): 277-298.

[17] Nicoud F, Benoit L, Sensiau C, Poinsot T, Acoustic modes in combustors with complex impedances and multidimensional active flames. AIAA Journal, 2007, 45(2): 426-441.

[18] Bigongiari A, Heckl MA, A Green's function approach to the rapid prediction of thermoacoustic instabilities in combustors. Journal of Fluid Mechanics, 2016, 798: 970-996.

[19] Pant $T$, Wang $H$, Transported PDF modeling of thermo-acoustic instability in a self-excited model rocket combustor using Eulerian Monte Carlo Fields method. AIAA SciTech 2019 Forum, 2019, pp. 1496.

[20] Kraus C, Selle L, Poinsot T, Coupling heat transfer and large eddy simulation for combustion instability prediction in a swirl burner. Combustion and Flame, 2018, 191: 239-251.

[21] Silva CF, Magri L, Runte T, Polifke W, Uncertainty quantification of growth rates of thermoacoustic instability by an adjoint Helmholtz solver. Journal of Engineering for Gas Turbines and Power, 2017, 139(1): 011901.

[22] Mejia D, Miguel-Brebion M, Selle L, On the experimental determination of growth and damping rates for combustion instabilities. Combustion and Flame, 
Dan Zhao et al. Thermodynamics-Acoustics Coupling Studies on Self-excited Combustion Oscillations Maximum Growth Rate 13

2016, 169: 287-296.

[23] Ghirardo G, Boudy F, Bothien MR, Amplitude statistics prediction in thermoacoustics. Journal of Fluid Mechanics, 2018, 844: 216-246.

[24] Bothien MR, Noiray N, Schuermans B, A novel damping device for broadband attenuation of low-frequency combustion pulsations in gas turbines. Journal of Engineering for Gas Turbines and Power, 2014, 136(4): 041504 .

[25] Noiray N, Denisov A, A method to identify thermoacoustic growth rates in combustion chambers from dynamic pressure time series. Proceedings of the Combustion Institute, 2017, 36(3): 3843-3850.

[26] Kim KT, Hochgreb S, Measurements of triggering and transient growth in a model lean-premixed gas turbine combustor. Combustion and Flame, 2012, 159(3): 1215-1227.

[27] Fleifil M, Annaswamy AM, Ghoneim ZA, Ghoniem AF, Response of a laminar premixed flame to flow oscillations: A kinematic model and thermoacoustic instability results. Combustion and Flame, 1996, 106(4): 487-510.

[28] Schulze M, Sattelmayer T, A comparison of time and frequency domain descriptions of high frequency acoustics in rocket engines with focus on dome coupling. Aerospace Science and Technology, 2015, 45: 165-173.

[29] Balusamy S, Li LK, Han Z, Hochgreb S, Extracting flame describing functions in the presence of self-excited thermoacoustic oscillations. Proceedings of the Combustion Institute, 2017, 36(3): 3851-3861.

[30] Juniper MP, Triggering in the horizontal Rijke tube: non-normality, transient growth and bypass transition. Journal Of Fluid Mechanics, 2011, 667: 272-308.

[31] Su W, Wang N, Li J, Zhao Y, Yan M, Improved method of measuring pressure coupled response for composite solid propellants. Journal of Sound and Vibration, 2014, 333(8): 2226-2240.

[32] Morgans AS, Dowling AP, Model-based control of combustion instabilities. Journal of Sound and Vibration, 2007, 299(1-2): 261-282.

[33] Blonbou R, Laverdant A, Zaleski S, Kuentzmann P, Active control of combustion instabilities on a Rijke tube using neural networks. Proceedings of the Combustion Institute, 2000, 28(1): 747-755.

[34] Bloxsidge GJ, Dowling AP, Langhorne PJ, Reheat buzz: an acoustically coupled combustion instability. Part 2 . Theory. Journal of Fluid Mechanics, 1988, 193: 445-473.

[35] Heckl MA, Active control of the noise from a Rijke tube. Journal of Sound and Vibration, 1988, 124(1): 117-133.

[36] Yu YC, Sisco JC, Rosen S, Madhav A, Anderson WE, Spontaneous longitudinal combustion instability in a continuously-variable resonance combustor. Journal of Propulsion and Power, 2012, 28(5): 876-887.
[37] Gulati A, Mani R, Active control of unsteady combustion-induced oscillations. Journal of Propulsion and Power, 1992, 8(5): 1109-1115.

[38] Burnley VS, Culick FE, Influence of random excitations on acoustic instabilities in combustion chambers. AIAA Journal, 2000, 38(8): 1403-1410.

[39] Eckstein J, Sattelmayer T, Low-order modeling of low-frequency combustion instabilities in aeroengines. Journal of Propulsion and Power, 2006, 22(2): 425432.

[40] Motheau E, Nicoud F, Poinsot T, Mixed acoustic-entropy combustion instabilities in gas turbines. Journal of Fluid Mechanics, 2014, 749: 542-576.

[41] Zhao D, Gutmark E, Reinecke A, Mitigating self-excited flame pulsating and thermoacoustic oscillations. Science Bulletin, 2019, 64: 941-952.

[42] Raun RL, Beckstead MW, Finlinson JC, Brooks KP, A review of Rijke tubs, Rijke burners and related devices. Progress in Energy and Combustion Science, 1993, 19: 313-364.

[43] Feldman Jr KT, Reivew of the literature on Rijke thermoacoustic phenomena. Journal of Sound and Vibration, 1968, 7(1): 83-89.

[44] A. P. Dowling, J. E. Ffowcs Williams, Sound and Sources of Sound. John Wiley \& Sons Inc, 2000, Chister, UK, pp. $18-23$.

[45] Hashimoto T, Shibuya H, Gotoda H, Ohmichi Y, Matsuyama S, Spatiotemporal dynamics and early detection of thermoacoustic combustion instability in a model rocket combustor. Physical Review E, 2019, 99 (3): 032208.

[46] Backhaus S, Swift GW, A thermoacoustic Stirling heat engine, Nature, 1999, 399 (6734): 335-338.

[47] Barinaga M, Acoustic fridge takes to space. Science, 1992, 255(5044): 534-535.

[48] Yazaki T, Sugioka S, Mizutani F, Mamada H, Nonlinear dynamics of a forced thermoacoustic oscillation. Physical review letters, 1990, 64(21): 2515.

[49] Kryuchkov NP, Yakovlev EV, Gorbunov EA, Couëdel L, Lipaev AM, Yurchenko SO, Thermoacoustic instability in two-dimensional fluid complex plasmas. Physical review letters, 2018, 121(7): 075003.

[50] Cengel YA, Boles MA, Thermodynamics: An engineering approach. $7^{\text {th }}$ Edition, McGraw Hill, New York, USA, 2011.

[51] Bai X, Cheng P, Li Q, Sheng L, Kang Z, Effects of self-pulsation on combustion instability in a liquid rocket engine. Experimental Thermal and Fluid Science, 2020, 114: 110038.

[52] Zhou H, Tao C, Liu Z, Meng S, Cen K, Optimal control of turbulent premixed combustion instability with annular micropore air jet. Aerospace Science and Technology, 2020, 98: 105650 . 